\title{
The Money Pump as a Measure of Revealed Preference Violations
}

\section{Federico Echenique}

California Institute of Technology

\section{Sangmok Lee}

California Institute of Technology

\section{Matthew Shum}

California Institute of Technology

\begin{abstract}
We introduce a measure of the severity of violations of the revealed preference axioms, the money pump index (MPI). The MPI is the amount of money one can extract from a consumer who violates the axioms. It is also a statistical test for the hypothesis that a consumer is rational when behavior is observed with error. We present an application using a panel data set of food expenditures. The data exhibit many violations of the axioms. Mostly, the MPI for these violations is small. The MPI indicates that the hypothesis of consumer rationality cannot be rejected.
\end{abstract}

\section{Introduction}

The assumption that consumers are rational is one of the oldest and most controversial assumptions in economics. Conceptually, the empirical content of the rationality assumption has been very well understood since the works of Samuelson (1938), Richter (1966), Afriat (1967), and

We thank Dan Silverman for comments and the editor and two anonymous referees for many useful suggestions on previous drafts. 
Varian (1982): revealed preference theory captures the empirical content of rational consumption behavior.

As a practical matter, however, revealed preference analysis is problematic because of the "all or nothing" nature of the exercise: either a data set satisfies the generalized axiom of revealed preference (GARP) or it does not. In practice, however, it is useful to gauge how severely consumers violate the axiom. Our paper presents a new measure of the severity of a violation of GARP. The measure is based on the idea that a consumer who violates GARP is subject to being exploited as a "money pump." We propose that the severity of a violation be measured by the amount of money that could be extracted from the consumer; we call this the money pump index (MPI).

The MPI implements a statistical test for the hypothesis of consumer rationality. A violation of GARP may be the result of measurement errors in prices or in consumer choices. The MPI measures how likely it is that a violation of GARP is due to such errors and essentially compatible with rational behavior.

We present an empirical application to household-level "scanner" panel data containing time series of household-level food grocery purchases collected at checkout scanners in supermarkets. In contrast to many earlier studies, using more aggregate or cross-sectional consumption data, our analysis revealed a substantial number of violations of GARP. Specifically, 396 out of the 494 households in our data set violate GARP at some point. However, most of these violations are not severe: our MPI is centered around 6 percent of a household's food expenditures, or about $\$ 12.80$ when evaluated at the average monthly food expenditure of $\$ 213$.

The magnitudes of the MPI appear intuitively very small (in both dollar and percentage terms). We proceed to formally use the MPI to test the hypothesis of consumer rationality. We test whether the MPI could be accounted for simply by measurement errors in the variables. In our empirical application, we are unable to reject the null hypothesis that the observed MPIs are consistent with rational behavior and measurement errors. In other words, the apparently small 6 percent MPI is also small in a statistical sense.

We correlate our measure with demographic variables. Most results are intuitive: less educated, poorer, and older households make more severe violations of GARP than highly educated, richer, and younger households do. On the other hand, smaller households make more severe violations of GARP. Moreover, because the demand for many food grocery items reflects seasonal trends, we also check whether GARP violations are more severe when comparing consumption between peak and nonpeak seasonal periods. We find no evidence of this, implying 

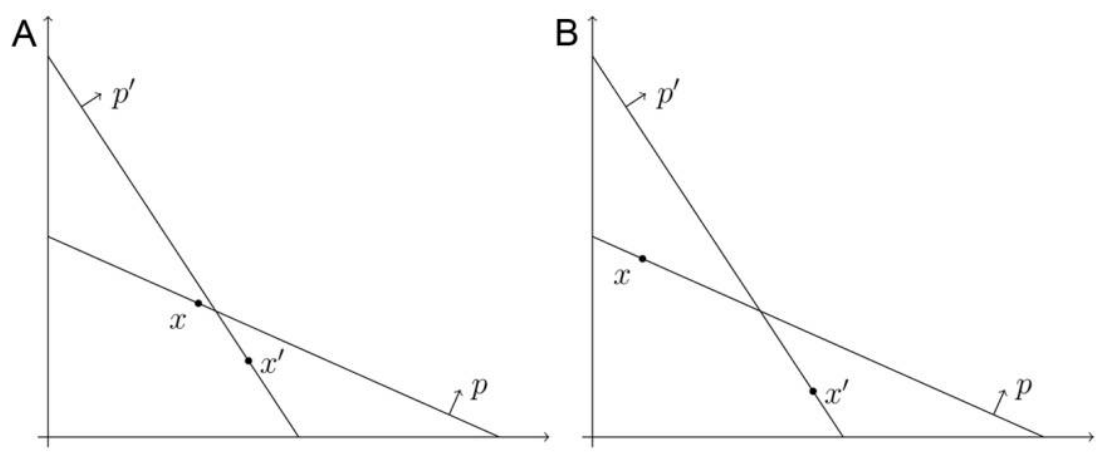

FIG. 1.-Two observations: $(x, p)$ and $\left(x^{\prime}, p^{\prime}\right) . A,(x, p)$ and $\left(x^{\prime}, p^{\prime}\right)$ violate GARP (in fact WARP). $B$, A more "severe" violation of GARP.

that consumption in our data can be modeled by stable preferences that exhibit no seasonal component.

Money pump.-Our measure of the severity of a GARP violation is motivated by the idea that a violation of GARP exposes a consumer to being manipulated as a "money pump." For example, consider the situation in figure $1 A$. A consumer buys bundle $x$ at prices $p$ and $x^{\prime}$ at prices $p^{\prime}$. Evidently, there is a violation of GARP (actually of WARP, the weak axiom of revealed preference) because $x$ was purchased when $x^{\prime}$ was affordable, and vice versa. Knowing these choices, a devious "arbitrager" who follows the opposite purchasing strategy (buying bundle $x$ at prices $p^{\prime}$ and bundle $x^{\prime}$ at prices $p$ ) could profitably resell $x$ to the consumer at prices $p$ and $x^{\prime}$ at prices $p^{\prime}$. The total profit the arbitrager would make equals

$$
m p=p \cdot\left(x-x^{\prime}\right)+p^{\prime} \cdot\left(x^{\prime}-x\right) .
$$

We use the magnitude $m p$, "money pump cost," to measure the severity of the violation of GARP. Specifically, our MPI is the money pump cost expressed as a percentage of expenditure.

The MPI is an intuitive measure of the severity of a violation of GARP. Consider the situations in figures $1 A$ and $B$. Each figure presents a violation of GARP, but intuitively the violation in panel $B$ is more severe than the one in panel $A$. The money pump cost reflects this difference. Figures $2 A$ and $B$ represent the money pump cost: it is the sum of the translation of the $p$-budget line (from crossing $x$ to crossing $x^{\prime}$ ) and the translation of the $p^{\prime}$-budget line (from crossing $x^{\prime}$ to crossing $x$ ). The money pump represents the severity of the violations, and it is expressed in monetary terms, so the numerical value of a violation has a clear 

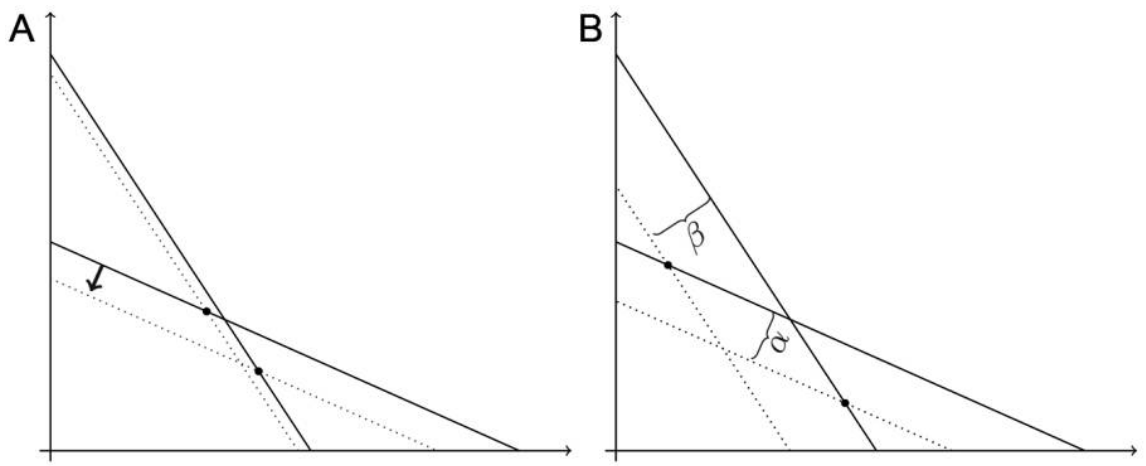

Fig. 2.-Money pump costs for figure 1. A, Two observations: $(x, p)$ and $\left(x^{\prime}, p^{\prime}\right) . B$, $m p=\alpha+\beta, \alpha=p \cdot\left(x-x^{\prime}\right), \beta=p^{\prime} \cdot\left(x^{\prime}-x\right)$.

interpretation. As we explain below, it also functions as a formal test statistic.

The idea that arbitragers can "pump money" from irrational consumers is not new, and it has been used as a reason for why one should not observe irrational behavior. For our purposes, however, the devious arbitrager is a fictional character. There is a debate on whether GARP violators would be driven out of the market because of the actions of arbitragers who exploit them as money pumps (see, e.g., Mulligan 1996; Rabin 2002; Laibson and Yariv 2007). We do not take a stand on the issue: our use of the money pump is purely an application of the idea captured by figure 1 .

Panel data.-Many of the recent studies of GARP employ repeated cross-sectional data; tests of GARP implemented on such data require some sort of aggregation or "matching" of similar households across different cross sections. In contrast, our panel data allow us to study how household purchases vary with prices over time without the need to aggregate or "match" consumers. By focusing on supermarket purchases, we also observe a higher frequency of price changes relative to expenditure, compared to standard cross-sectional consumption data sets. As is well known (see, e.g., Blundell, Browning, and Crawford 2003), a large variability in expenditure relative to prices can result in GARP having low power.

\section{Related Literature}

The literature on testing the revealed preference axioms is large and contains both classical papers and more recent contributions. Afriat (1967) and Varian (1982) are seminal contributions to the methodology 
of revealed preference tests; Varian (2006) provides a survey. Empirical applications of revealed preference tests have employed both field and experimental data.

In principle, tests of WARP/GARP require repeated observations of a decision-making unit (individual or household) across different pricing regimes. However, many of the empirical investigations of GARP using field data employ data from cross-sectional household-level surveys (such as the Consumer Expenditure Survey in the United States and the Family Expenditure Survey in the United Kingdom). Thus, an important challenge addressed in these papers is how to "match" households across different time periods to form a synthetic panel. Blundell et al. (2003) and Blundell, Chen, and Kristensen (2007) address this issue by estimating an "Engel curve" relating a household's consumption to prices, expenditure, and household demographics, and they test GARP by comparing the predicted consumption behavior of households with similar demographics and expenditure levels across different pricing regimes. Hoderlein and Stoye (2009) take a more agnostic approach and use results from the copula literature to obtain bounds on the percentage of households that violate WARP in two separate cross sections of survey data.

In the present paper, we avoid these difficulties by using a long household-level scanner panel data set, where the purchase decisions of given households over a 2-year period are observed. To our knowledge, testing the revealed preference axioms using scanner data is new in the literature.

At the same time, a large literature testing revealed preference using experimental data has also developed. This literature has employed both laboratory experiments (recent contributions include Sippel [1997], Andreoni and Miller [2002] and Fevrier and Visser [2004]) and field experiments utilizing unique subject pools (psychiatric patients in Battalio et al. [1973], children in Harbaugh, Krause, and Berry [2001], and tufted capuchin monkeys in Chen, Lakshminarayanan, and Santos [2006]).

It is fair to say that most of the empirical literature, using both field and experimental data, finds relatively few violations of GARP. Therefore, the power of GARP as a test of rationality is a real concern; these issues have been discussed in, among others, Bronars (1987), Blundell et al. (2003), Andreoni, Gillen, and Harbaugh (2011), and Beatty and Crawford (2011). Experiments suffer less from this problem because they are often carefully designed to avoid power issues (see, e.g., Andreoni and Miller 2002).

At the same time, revealed preference tests are quite stark, allowing for either rational or irrational consumers. In practice, one would like to accommodate a grey area in which "small" violations of GARP may 
not indicate a worrying degree of irrationality (or may indicate imperfections in the data). In the existing literature, various researchers have proposed ways to quantify the degree of violations from GARP, including Afriat (1967), Varian (1985, 1990), Gross (1995), and Heufer (2008). ${ }^{1}$

In terms of assessing the severity of violations of GARP, MPI is closest in spirit to the efficiency index originally proposed by Afriat (1967) and subsequently modified by Varian (1990). Jerison and Jerison (2011) is a recent contribution, relating Afriat's efficiency index to a measure of the asymmetry of the Slutsky matrix. Choi et al. (2011) use Afriat's index on data from a large-scale field experiment; they find some of the same qualitative empirical results as we do using MPI on scanner data (specifically the results we report in Sec. IV.C). We review these developments in Section III.B below.

\section{Methodology}

\section{A. Money Pump Index}

Suppose that we observe the purchases of a single consumer when she faces different prices. Observation $k(k=1, \ldots, K)$ consists of a consumption bundle $x^{k} \in \mathbb{R}_{+}^{l}$ that the consumer bought at prices $p^{k} \in$ $\mathbb{R}_{++}^{l}$.

Let $X$ be the set of all observed consumption bundles; that is, $X=$ $\left\{x^{k}: k=1, \ldots, K\right\}$. The revealed preference relation on $X$ is the binary relation $R$ defined as $x^{k} R x^{l}$ if $p^{k} \cdot x^{k} \geq p^{k} \cdot x^{l}$. The strict revealed preference relation is the binary relation $P$ defined as $x^{k} P x^{l}$ if $p^{k} \cdot x^{k}>$ $p^{k} \cdot x^{l}$.

The data satisfy the weak axiom of revealed preference (WARP) if whenever $x^{k} R x^{l}$ it is false that $x^{l} P x^{k}$.

The data satisfy the generalized axiom of revealed preference (GARP) if there is no sequence $x^{k_{1}}, x^{k_{2}}, \ldots, x^{k_{n}}$ such that

$$
x^{k_{1}} R x^{k_{2}} R, \ldots, R x^{k_{n}} \text { while } x^{k_{n}} P x^{k_{1}} .
$$

A violation of GARP is identified with a sequence $x^{k_{1}}, x^{k_{2}}, \ldots, x^{k_{n}}$. We say that $n$ is the length of the sequence.

Given a sequence $x^{k_{1}}, x^{k_{2}}, \ldots, x^{k_{n}}$ for which (1) holds, we can compute the money pump cost associated to this sequence as

$$
\sum_{l=1}^{n} p^{k_{l}} \cdot\left(x^{k_{l}}-x^{k_{l+1}}\right)
$$

(taking $\left.k_{n+1}=k_{1}\right)$.

\footnotetext{
${ }^{1}$ Apesteguia and Ballester (2010) axiomatize a measure of deviations from rationality. It applies in general choice environments with finitely many choices. It does not use the special structure of Walrasian budgets.
} 
Our money pump cost is measured in dollars. In order to compare this cost across consumers with different budgets, we normalize the cost by each household's total expenditure. Specifically, the MPI equals the money pump cost as a proportion of total expenditure: if (1) holds for the sequence $x^{k_{1}}, x^{k_{2}}, \ldots, x^{k_{n}}$, we compute the MPI of the sequence as

$$
\operatorname{MPI}_{\left\{\left(x^{k_{1}}, p^{k_{1}}\right), \ldots,\left(x^{k_{n},}, p^{\left.\left.k_{n}\right)\right\}}\right.\right.}=\frac{\sum_{l=1}^{n} p^{k_{l}} \cdot\left(x^{k_{l}}-x^{k_{l+1}}\right)}{\sum_{l=1}^{n} p^{k_{l}} \cdot x^{k_{l}}}
$$

(taking $\left.k_{n+1}=k_{1}\right)$.

Note that MPI is measured for each violation of GARP. In the empirical application in Section IV.B, we sometimes report a householdlevel MPI by computing the mean and median MPI across the different violations of GARP for a given household.

Remark 1. Calculating money pump costs can be a huge computational task. For the data we present in Section IV, $K=26$, so there are

$$
\sum_{k=2}^{26}\left(\begin{array}{c}
26 \\
k
\end{array}\right)(k-1) ! \approx 4.39239 \times 10^{25}
$$

potential cycles, which are unique up to rotations. There are fast algorithms for checking if GARP has been violated (see Varian 1982), but they do not suffice to calculate MPI. ${ }^{2}$

\section{B. Comparison with Afriat's Efficiency Index}

We briefly review and compare our approach to the "efficiency indices" proposed by Afriat (1967) and Varian (1990) to quantify violations from GARP. Given $e \in[0,1]$, let $R_{e}$ and $P_{e}$ be the binary relations defined by $x^{k} R_{e} x^{l}$ if $e p^{k} \cdot x^{k} \geq p^{k} \cdot x^{l}$ and $x^{k} P_{e} x^{l}$ if $e p^{k} \cdot x^{k}>p^{k} \cdot x^{l}$. Clearly, if $e=1$, then $R_{e}$ is the original revealed preference relation; so if $R_{1}$ satisfies GARP, then the data are consistent with rationality. At the other extreme, $R_{0}$ satisfies GARP trivially. Afriat's efficiency index (AEI) is defined as the supremum over all the numbers $e$ such that $\left(R_{e}, P_{e}\right)$ satisfies GARP.

The ideas behind AEI are similar to our MPI (perhaps unavoidably so, as they try to measure the same phenomenon), but AEI and MPI differ in their interpretations. MPI is the monetary magnitude that can be extracted from a consumer that violates GARP. AEI can be interpreted as a "margin of error" (Varian 1990) that we allow the agent to make in his consumption choices or as a tolerance for wasted expenditure.

The behaviors of the MPI and AEI can be quite different, and they

${ }^{2}$ Warshall's algorithm, suggested by Varian for checking GARP, can be used to calculate an approximation of MPI. 


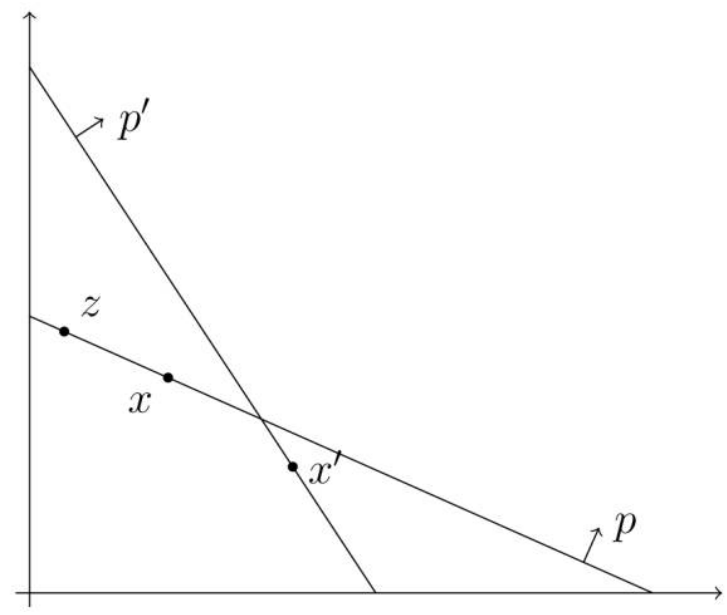

FIG. 3.-Two violations of WARP: $(x, p),\left(x^{\prime}, p^{\prime}\right)$ and $(z, p),\left(x^{\prime}, p^{\prime}\right)$

can give opposite conclusions on the same data. We present two simple examples to illustrate this point. The first example is in figure 3 , which shows two pairs of observations $\left\{(z, p),\left(x^{\prime}, p^{\prime}\right)\right\}$ and $\left\{(x, p),\left(x^{\prime}, p^{\prime}\right)\right\}$, which both violate WARP. The MPIs for these observations are

$$
\begin{aligned}
\operatorname{MPI}_{\left\{(z, p),\left(x^{\prime}, p^{\prime}\right)\right\}}= & \frac{p \cdot\left(z-x^{\prime}\right)+p^{\prime} \cdot\left(x^{\prime}-z\right)}{p \cdot z+p^{\prime} \cdot x^{\prime}} \\
& >\frac{p \cdot\left(x-x^{\prime}\right)+p^{\prime} \cdot\left(x^{\prime}-x\right)}{p \cdot x+p^{\prime} \cdot x^{\prime}}=\operatorname{MPI}_{\left\{(x, p),\left(x^{\prime}, p^{\prime}\right)\right\}},
\end{aligned}
$$

since, as drawn in figure $3, p \cdot x=p \cdot z$ and $p^{\prime} \cdot\left(x^{\prime}-z\right)>p^{\prime} \cdot\left(x^{\prime}-x\right)$. We conclude that the violation in the data $(z, p),\left(x^{\prime}, p^{\prime}\right)$ is more severe than in $(x, p),\left(x^{\prime}, p^{\prime}\right)$.

Calculating the AEI for these pairs of violations yields

$$
\begin{aligned}
\operatorname{AEI}_{\left\{(z, p),\left(x^{\prime}, p^{\prime}\right)\right\}} & =\max \left\{\frac{p^{\prime} \cdot z}{p^{\prime} \cdot x^{\prime}}, \frac{p \cdot x^{\prime}}{p \cdot z}\right\}=\frac{p \cdot x^{\prime}}{p \cdot z} \\
& =\frac{p \cdot x^{\prime}}{p \cdot x}=\max \left\{\frac{p^{\prime} \cdot x}{p^{\prime} \cdot x^{\prime}}, \frac{p \cdot x^{\prime}}{p \cdot x}\right\}=\operatorname{AEI}_{\left\{(x, p),\left(x^{\prime}, p^{\prime}\right)\right\}} ;
\end{aligned}
$$

that is, the AEIs are the same for both violations.

As the example illustrates, the crucial difference between AEI and MPI lies in how it treats each difference $p^{k} \cdot x^{k}-p^{k} \cdot x^{l}$ in a violation of GARP. The MPI simply adds up the differences, and the resulting measure of a violation of GARP is the dollar amount that can be extracted by running the money pump implied by the violation of GARP. 
The AEI, on the other hand, seeks to "break" the violation of GARP at its weakest link. Thus, once $e<1$ deflates a value of expenditure $p^{k} \cdot x^{k}$ to the point where there is no violation of GARP, the remaining differences $p^{k} \cdot x^{k}-p^{k} \cdot x^{l}$ play no role in the measure. ${ }^{3}$

We illustrate the point with a second concrete example. The example exhibits a small violation of GARP according to AEI but a large one according to MPI. In fact, the violation is negligible according to AEI, but the parameter $\delta$ below can be chosen so that MPI is arbitrarily large. Let $p^{1}=\left(\frac{1}{\delta}, \delta\right), p^{2}=\left(\delta, \frac{1}{\delta}\right), p^{3}=(1,1), x^{1}=\left(\delta^{2}, 0\right)$,

$$
x^{2}=\left(\frac{\delta^{2}}{1+\delta^{2}}, \frac{\delta^{2}}{1+\delta^{2}}\right),
$$

and $x^{3}=(1,0)$. Then $p^{1} \cdot x^{1}=p^{1} \cdot x^{2}=\delta, p^{2} \cdot x^{2}=p^{2} \cdot x^{3}=\delta$, $p^{3} \cdot x^{3}=1$, and $p^{3} \cdot x^{1}=\delta^{2}$. So the $e$-revealed preference relation $R_{e}$ satisfies GARP for any $e<1$; AEI therefore equals one. ${ }^{4}$ On the other hand,

$$
\begin{aligned}
\text { MPI }= & \left\{\left(\frac{1}{\delta}, \delta\right)\left[\left(\delta^{2}, 0\right)-\left(\frac{\delta^{2}}{1+\delta^{2}}, \frac{\delta^{2}}{1+\delta^{2}}\right)\right]+\left(\delta, \frac{1}{\delta}\right)\right. \\
& \left.\cdot\left[\left(\frac{\delta^{2}}{1+\delta^{2}}, \frac{\delta^{2}}{1+\delta^{2}}\right)-(1,0)\right]\right\} \div(\delta+\delta+1) \\
& +\frac{(1,1) \cdot\left[(1,0)-\left(\delta^{2}, 0\right)\right]}{\delta+\delta+1},
\end{aligned}
$$

which approaches one as $\delta \rightarrow 0$. So if $\delta$ is small, MPI exhibits a large violation of GARP, whereas the violation is mild according to AEI. Thus the AEI and MPI can give the opposite conclusion on the same data.

The observations also suggest that MPI and AEI are more likely to differ on longer sequences that violate GARP than on shorter sequences. As we shall see in Section IV.B, that is indeed the case in our data.

\section{Statistical Tests Using the MPI: How Large Is "Large"?}

We formulate a statistical basis for testing whether a violation of WARP could be explained by either simple mistakes on the part of the con-

\footnotetext{
${ }^{3}$ Varian modifies AEI by allowing $e$ to vary across the different price vectors, looking at a vector $\left(e_{k}\right)$. Varian's measure is the closest distance to the unit vector $\left(e_{k}=1\right)$ of an $\left(e_{k}\right)$ with no violations of GARP. This version of AEI suffers from the same problem. When we minimize the distance $\left\|\left(e_{k}\right)-\mathbf{1}\right\|$, if we fix the value of one $e_{k}$ such that there is no violation of GARP, we want to set the other entries in the vector equal to one.

${ }^{4}$ Similarly, Varian's version of AEI would count these data as basically consistent with GARP, as there are vectors $\left(e^{1}, e^{2}, e^{3}\right)$ arbitrarily close to $(1,1,1)$ such that the corresponding relations satisfy GARP.
} 
sumer or measurement errors in variables. Such an approach to testing WARP was pioneered by Varian $(1985,1990)$.

We assume that there is measurement error in prices such that $p=$ $q+\varepsilon$, where $p$ are observed prices, $q$ are true but unobserved (latent) prices, and $\varepsilon$ is an independent and identically distributed measurement error drawn from a normal distribution with mean $\mu$ and variance $\sigma^{2}$, independently across households and prices. The assumption that observed prices contain measurement error is natural for our empirical application below because differences between shelf prices (which are observed) and transactions prices (which we do not completely observe) constitute the most important source of measurement error in supermarket data sets. ${ }^{5}$

Recall that the money pump cost for observations $\left(\left(p^{1}, x^{1}\right),\left(p^{2}\right.\right.$, $\left.x^{2}\right)$ ) violating WARP is defined as

$$
\begin{aligned}
\hat{T}_{M P} \equiv & {\left[\left(p^{1}-p^{2}\right) \cdot\left(x^{1}-x^{2}\right)\right] \mathbf{1}_{\left\{\left(\left(p^{1}, x^{1}\right),\left(p^{2}, x^{2}\right)\right) \text { violate WARP }\right\}} } \\
= & {\left[\left(q^{1}-q^{2}\right) \cdot\left(x^{1}-x^{2}\right)+\left(\varepsilon^{1}-\varepsilon^{2}\right)\right.} \\
& \left.\cdot\left(x^{1}-x^{2}\right)\right] \mathbf{1}_{\left\{\left(\left(q^{1}+\varepsilon^{1}, x^{1}\right),\left(q^{2}+\varepsilon^{2}, x^{2}\right)\right) \text { violate WARP }\right\}} .
\end{aligned}
$$

Even with the normality assumption on the measurement errors $(\varepsilon)$, we cannot derive the distribution of $\hat{T}_{M P}$ under the null of rationality, that is, that $\left(q^{1}, x^{1}\right),\left(q^{2}, x^{2}\right)$ satisfy WARP and that the observed violation of WARP was generated only because of measurement error in prices. However, taking a cue from Varian (1985), we can bound $\hat{T}_{M P}$ by another random variable $T_{M P}$ and perform the desired hypothesis test based on the distribution of $T_{M P}$ rather than $\hat{T}_{M P}$. Specifically, because $\left(p^{1}-\right.$ $\left.p^{2}\right) \cdot\left(x^{1}-x^{2}\right) \geq 0$ when $\left(\left(p^{1}, x^{1}\right),\left(p^{2}, x^{2}\right)\right)$ violates WARP, we have

$$
\begin{aligned}
\hat{T}_{M P}= & {\left[\left(q^{1}-q^{2}\right) \cdot\left(x^{1}-x^{2}\right)+\left(\varepsilon^{1}-\varepsilon^{2}\right)\right.} \\
& \left.\cdot\left(x^{1}-x^{2}\right)\right] \mathbf{1}_{\left\{\left(\left(q^{1}+\varepsilon^{1}, x^{1}\right),\left(q^{2}+\varepsilon^{2}, x^{2}\right)\right) \text { violate WARP }\right\}} \\
= & \max \left\{0,\left(q^{1}-q^{2}\right) \cdot\left(x^{1}-x^{2}\right)+\left(\varepsilon^{1}-\varepsilon^{2}\right) \cdot\left(x^{1}-x^{2}\right)\right\} .
\end{aligned}
$$

To proceed further, we require an additional assumption. Suppose that we are given consumption bundles $x^{1}$ and $x^{2}$ that were chosen at some observed prices, $p^{1}$ and $p^{2}$, respectively. Let us assume that $x^{1}$ and $x^{2}$ are rational choices at the true (but unobserved) prices, say $q^{1}$ and $q^{2}$. Then it follows from Afriat's theorem (see Afriat 1967; Varian 1985) that there are positive numbers $\lambda^{1}$ and $\lambda^{2}$ such that

$$
\lambda^{1} q^{1} \cdot\left(x^{1}-x^{2}\right)+\lambda^{2} q^{2} \cdot\left(x^{2}-x^{1}\right) \leq 0 .
$$

\footnotetext{
${ }^{5}$ Varian (1985) assumed measurement error in choices rather than in prices. This difference is insubstantial, as the test we describe below would also work for errors in choices. For our empirical application, assuming measurement error in prices seems more natural.
} 
The numbers $\lambda^{1}$ and $\lambda^{2}$ have a natural interpretation as Lagrange multipliers. They are the marginal utility of a relaxation of the budget constraint, the "marginal utility of income." We make an additional assumption that the two marginal utilities are equal.

Assumption. Equal marginal utility of income (EMUI): $\lambda^{1}=\lambda^{2}$.

The EMUI assumption has a clear economic meaning: at the point at which the purchases were made, an additional dollar in income would result in the same increase in utility. It seems a reasonable assumption for our application to supermarket purchases. Assuming EMUI, we obtain that

$$
\left(q^{1}-q^{2}\right) \cdot\left(x^{1}-x^{2}\right) \leq 0 .
$$

Using this inequality in equation (3), we get

$$
\begin{aligned}
\hat{T}_{M P} & =\max \left\{0,\left(q^{1}-q^{2}\right) \cdot\left(x^{1}-x^{2}\right)+\left(\varepsilon^{1}-\varepsilon^{2}\right) \cdot\left(x^{1}-x^{2}\right)\right\} \\
& \leq \max \left\{0,\left(\varepsilon^{1}-\varepsilon^{2}\right) \cdot\left(x^{1}-x^{2}\right)\right\} \equiv T_{M P} .
\end{aligned}
$$

The upshot is that the observed money pump cost $\hat{T}_{M P}$ can be bounded from above by $T_{M P}$, which, given the distributional assumptions on $\varepsilon$, follows a truncated normal distribution with mean zero and variance $2 \cdot\left\|x^{1}-x^{2}\right\|^{2} \cdot \sigma^{2}$.

Given a nominal size $\alpha$, then, we can find a critical value $C_{\alpha}$ satisfying $P\left(T_{M P}>C_{\alpha}\right)=\alpha$; we set $C_{\alpha}=F_{T_{M P}}^{-1}(1-\alpha)$, where $F_{T_{M P}}$ denotes the cumulative distribution function of $T_{M P}$. However, because $\hat{T}_{M P} \leq T_{M P}$, the "true size" of the test is

$$
P\left(\hat{T}_{M P}>C_{\alpha}\right) \leq P\left(T_{M P}>C_{\alpha}\right)=\alpha,
$$

so there is a downward size distortion: When MPI is large enough to warrant a rejection, we can do so with at least the desired confidence $1-\alpha$. However, the test tends to underreject, which raises power problems. We return to the power issue below in discussing the empirical application.

Tests for longer cycles.-We focused on a test for violations of WARP, but the same calculation holds for testing general violations of GARP. The condition derived from Afriat's theorem holds for general sequences of demands. Given $K$ observations of consumptions and prices and generalizing the EMUI assumption accordingly, we obtain

$$
\begin{aligned}
\hat{T}_{M P} & =\left[\sum_{k=1}^{K} p^{k} \cdot\left(x^{k}-x^{k+1}\right)\right] \mathbf{1}_{\left\langle\left(\left(p^{k}, x^{k}\right)\right)_{k=1}^{K}\right. \text { violates GARP }} \\
& \leq \max \left\{0, \sum_{k=1}^{K} \varepsilon^{k} \cdot\left(x^{k}-x^{k+1}\right)\right\} \equiv T_{M P},
\end{aligned}
$$


where $T_{M P}$ follows a truncated normal with mean zero and variance $\sum_{k=1}^{K}\left\|x^{k}-x^{k+1}\right\|^{2} \cdot \sigma^{2}$.

\section{Empirical Results: Incidence and Severity of GARP Violations}

\section{A. Data Description}

We use a household-level scanner panel data set, the so-called Stanford Basket Dataset, which contains grocery expenditure data for 494 households from four grocery stores in an urban area of a large U.S. midwestern city, between June 1991 and June 1993 (104 weeks). This data set was collected by Information Resources, Inc. and has also been used in, among others, Bell and Lattin (1998), Shum (2004), and Hendel and Nevo (2006a, 2006b).

We focus in this paper on households' expenditures on food categories, of which there are 14: bacon, barbecue, butter, cereal, coffee, crackers, eggs, ice cream, nuts, analgesics, pizza, snacks, and sugar. We restrict attention to food because we do not expect consumers to change their food expenditure very dramatically in response to changes in income; most foods are basic necessities, and the role for "luxurious" spending on food is arguably more limited than for other types of goods. ${ }^{6}$

We observe 103,345 transactions of 4,082 unique items, that is, unique Universal Product Codes (UPC). Each transaction records the household identity, UPC, transaction week, consumed units, price per unit (shelf prices), and the coupon value (if used).

For the prices in the empirical analysis, we used the "shelf prices," which are the prices posted in the supermarket at the time of purchase. While the majority ( 86 percent) of transactions take place at shelf prices, the actual transaction price may differ from the shelf price, primarily because of the household's use of coupon discounts. We did not incorporate coupons into our analysis explicitly because we observe coupons only when a household uses them and do not observe coupon availability when either an item is not purchased or the coupon is not used. This partial observability of coupon discounts is problematic for revealed preference analysis because the GARP inequalities depend on a counterfactual calculation: how much would a consumption basket

\footnotetext{
${ }^{6}$ By focusing on food expenditures, our approach requires an assumption that food items are separable in households' preferences, so that purchases of nonfoods affect food consumption only through the income left over from such purchases. Hence, our test is implicitly a joint test of rationality and separability for food. However, separability is ubiquitous as an assumption in applied demand analysis and has been universally assumed in applied work to reduce the dimensionality of demand system (a point emphasized by Deaton and Muellbauer [1980] and Blundell [1988], among others).
} 
cost at prices at which another consumption basket was purchased? ${ }^{7}$ For these reasons, we use shelf prices in our analysis and consider coupon discounts as the primary source of measurement error in the price data.

In order to obtain consistent consumption data over goods, we aggregate transactions by brand name and category: when distinct items have a common brand name, their transactions are aggregated. Hence, each "product" in the sample is a food product with a distinct brand name, and we aggregate across all sizes/presentational forms of each product. Analogously, aggregate prices and discounts at the product level are obtained by averaging the prices and discounts of each size, weighted by the amount consumed. To minimize stockpiling and inventory issues, we also aggregate households' expenditures for each good over time to a 4-week period.

Even after this aggregation, not all brands are consumed for every time period; some products enter, some exit, and many others are simply unpopular items that are infrequently purchased by the households in the data set. Since GARP requires price observations over every time period, we use only brands for which price data are available for every period. For this reason, we drop 12,976 (or 12.5 percent) of the purchases from the data set.

\section{B. GARP and MPI}

Exploiting the panel nature of our data set, we next consider GARP violations for each household separately. This allows for arbitrary unobserved heterogeneity at the household level. The MPI is measured for each violation of GARP; we consider the mean or the median MPI across the household's violations.

Table 1 presents a summary of our results. Out of 494 households, 395 (roughly 80 percent) of them violate WARP (GARP for sequences of length 2) for at least some pairs of observations. Hence, a significant proportion of households do exhibit violations of WARP; our result is in contrast to much of the previous empirical literature, which fails to find many violations. Given remark 1, we check only for violations of GARP that involve cycles of limited length: lengths 2, 3, and 4 .

In table 1, moving from left to right, we include progressively longer cycles in testing GARP. When we include cycles of lengths 3 and 4, thereby searching a substantially larger number of possible cycles $(5,525$ and 95,225 , compared to 325$)$, the overall number of households violating GARP increases only by one (from 395 to 396). In theory GARP may be violated when WARP is satisfied. However, table 1 shows that

${ }^{7}$ Moreover, there are a few cases in which discounts (coupon values per unit) exceed shelf prices. 
TABLE 1

Money Pump: Calculated by Equation (2), Averaged over Households VIOLATING GARP

\begin{tabular}{lccc}
\hline \hline & \multicolumn{3}{c}{ CyCLE LeNGTHS INCLUdED } \\
\cline { 2 - 4 } & $\{2\}$ & $\{2,3\}$ & $\{2,3,4\}$ \\
\hline Total number of households & 494 & 494 & 494 \\
Households violating GARP & 395 & 396 & 396 \\
Median $^{\mathrm{a}}$ MPI & $5.97 \%$ & $5.95 \%$ & $5.91 \%$ \\
Mean $^{\mathrm{a}}$ MPI & $6.22 \%$ & $6.12 \%$ & $6.09 \%$ \\
Median $^{\mathrm{b}}(1-\mathrm{AEI})$ & $2.40 \%$ & $2.09 \%$ & $2.03 \%$ \\
Mean $^{\mathrm{b}}(1-\mathrm{AEI})$ & $2.63 \%$ & $2.38 \%$ & $2.31 \%$ \\
Possible cycles $_{\text {Median number of violations }}$ & 325 & 5,525 & 95,225 \\
Mean number of violations & 2 & 3 & 3 \\
\hline
\end{tabular}

${ }^{a}$ Numbers in these rows are the median/mean MPIs across all violations of GARP among the sample households.

${ }^{\mathrm{b}}$ Numbers in these rows are the median/mean values of $(1-\mathrm{AEI})$ across all violations of GARP among the sample households.

WARP closely approximates GARP in practice; only one household satisfies WARP while violating GARP. Moreover, the median and mean levels of MPI change only slightly as we search over longer cycles.

On the other hand, the severity of the violations, in terms of MPI (see eq. [2]), is moderate or small: the mean and median MPIs, taken across all households, are only about 6 percent of total expenditure.

To break this down further, we calculated, for each household, the MPI of each violation of WARP and obtained the household-specific median level of MPI, across all the cycles for this household, which violated WARP. In figure 4, we plot the cumulative distribution function of this household-specific median MPI across the 395 households that exhibit some violation of WARP. The function clearly rises very steeply for values of the MPI less than 10 percent but is largely flat thereafter. This indicates that a large majority of households have very small violations of WARP, and only a handful of households have larger violations, exceeding 10 percent of expenditures. Thus, large violations do occur, but they are infrequent.

Are these violations of GARP severe? - The finding that MPI is small is reinforced by the results of using MPI as a statistical test; we follow the method described in Section III.C. The observed standard deviation of price discounts by coupons is $\hat{\sigma}=1.1143$ (measured in cents per unit). Taking this as the value for the standard deviation in measurement error, and at a nominal size of $\alpha=5$ percent, we find no rejections of GARP at all; that is, none of the observed MPIs is large enough to warrant a rejection at this nominal size.

Since, by construction, our test will underreject relative to the nominal 


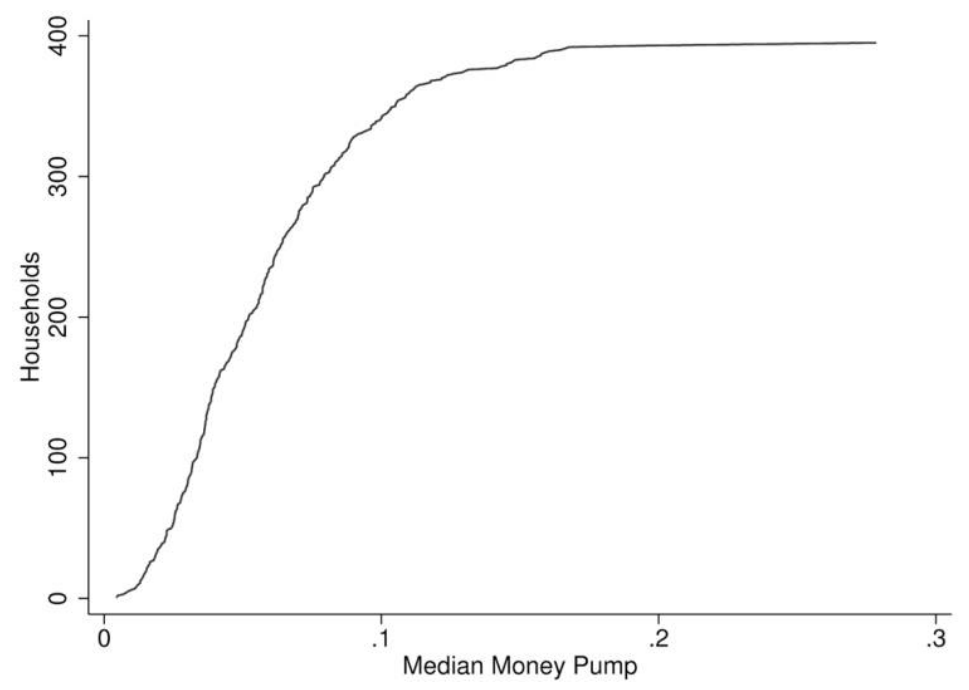

FIG. 4.-Cumulative distribution of households' median MPI

size, we reduced the value we used for the standard deviation of measurement error even further. Only by reducing this standard deviation by a factor of 5 , to $\frac{1}{5} \hat{\sigma}=0.2229$, are we able to reject around 5 percent of the observed MPIs. Hence, the value of $\sigma$ that we would need in order to reject GARP at usual significance levels is substantially lower than the standard deviation of price discounts in our data, which is a reasonable proxy for measurement error. Despite the tendency of our procedure to underreject, this is rather convincing evidence that the MPI fails to reject GARP.

Table 1 also exhibits an empirical comparison of MPI with AEI. A large value of AEI indicates a small violation, so we report instead the values of 1 - AEI. The correlation between MPI and $1-$ AEI may be instructive. The correlation is .7834, .6250, and .5197 for cycles of length 2, 3, and 4, respectively. As suggested by our discussion in Section III.B, the AEI and MPI differ more the longer the length of the cycle in the violation of GARP. ${ }^{8}$

\section{Demographic Variables}

Next, we consider the demographic determinants of rational (or irrational) consumption behavior, as measured by the MPI. Table 2 shows

\footnotetext{
${ }^{8}$ Using an approximation, we can compute these correlations for longer cycles. The correlation continues to decrease: .24 and -.44 for cycles of length 5 and 6 , respectively. The negative correlation at length 6 is purely driven by two specific observations.
} 
TABLE 2

Demographic Variables

\begin{tabular}{lc}
\hline \hline Variable & $\begin{array}{c}\text { Number of } \\
\text { Households }\end{array}$ \\
\hline Family size: & 187 \\
$\quad$ Mid size $(3,4$ members $)$ & 65 \\
$\quad$ Large size $(>4$ members $)$ & \\
Income: & 200 \\
$\quad$ Mid annual income ( $\in[\$ 20,000$, & 141 \\
$\quad \$ 45,000])$ & \\
$\quad$ High annual income $(>\$ 45,000)$ & 157 \\
Age: ${ }^{2}$ & 197 \\
$\quad$ Middle age & 255 \\
$\quad$ Old age & 480 \\
Education: & \\
$\quad$ High school & College \\
Total households & \\
\hline & a Middle-aged households are those in which the average of \\
the spouses' ages is between 30 and $65 ;$ in old-aged households, \\
this average exceeds 65.
\end{tabular}

the population distributions of the demographic variables. ${ }^{9}$ The panelists are generally older, and their education levels are higher than the general U.S. population.

Since MPI has a positive value only when consumptions violate WARP, we consider censored Tobit regressions of MPI on demographic variables. Table 3 shows the regression results with $156,000(=480 \times$ 325) observations: 480 households with $\left(\begin{array}{c}26 \\ 2\end{array}\right)$ possible pairs.

MPI is higher for older, poorer, and less educated households than for younger, richer, and more educated households. MPI is also higher for smaller households.

\section{Further Results}

\section{A. Seasonality and Stability of Preferences}

A consumer may fail GARP because his preferences change: they are not "stable." Given two observations, $(x, p)$ and $\left(x^{\prime}, p^{\prime}\right)$, it is possible that $x$ was a rational choice for a different utility function than $x^{\prime}$. We argue that unstable preferences would be reflected in a large MPI. Therefore, our empirical findings support the hypothesis that preferences are stable.

${ }^{9}$ The demographic data are missing for 14 households. We drop these from our data set and work with 480 households. 
TABLE 3

MPI Explained by Demographic Variables: Results from Tobit (Censored) REGRESSIONS

\begin{tabular}{lccc}
\hline \hline Variable & Regression 1 & Regression 2 & Regression 3 \\
\hline Middle age & & .0112 \\
& & $(1.92)$ \\
Old age & & .0126 \\
Mid family & $-.0161^{* * *}$ & $-.0118^{*}$ & $(1.74)$ \\
& $(-3.55)$ & $(-2.49)$ & -.0085 \\
Large family & $-.0281^{* * *}$ & $-.0243^{* * *}$ & $(-1.66)$ \\
& $(-4.04)$ & $(-3.45)$ & $-.0190^{*}$ \\
Middle income & & $-.0181^{* * *}$ & $(-2.52)$ \\
& & $(-3.44)$ & $-.0169^{* *}$ \\
High income & $-.0154^{*}$ & $(-3.02)$ \\
& & $(-2.57)$ & $-.0142^{*}$ \\
High school & -.0154 & -.00874 & $(-2.16)$ \\
& $(-1.84)$ & $(-1.02)$ & -.00768 \\
College & -.0162 & -.00666 & $(-.89)$ \\
& $(-1.95)$ & $(-.76)$ & -.00441 \\
Constant & $-.452^{* * *}$ & $-.450^{* * * *}$ & $(-.49)$ \\
& $(-30.63)$ & $(-30.57)$ & $-.463^{* * *}$ \\
$\sigma^{\mathrm{a}}$ & $.195^{* * *}$ & $.195^{* * *}$ & $(-27.56)$ \\
& $(36.65)$ & $(36.66)$ & $.195^{* * *}$ \\
Observations & 156,000 & 156,000 & $(36.66)$ \\
& & & 156,000 \\
\hline
\end{tabular}

NoTE.— $t$ statistics are in parentheses.

${ }^{\text {a }}$ Estimated standard deviation of errors in Tobit regression.

$* p<.05$

$* * p<.01$.

$* * * \quad p<.001$.

Consider a consumer who uses one utility for some purchases and another utility for other purchases. We argue that this consumer's MPI is positive for arbitrarily small changes in prices. In fact, MPI is larger when the difference in demands under both utilities is larger, thus implying that when preferences are unstable, MPI can be interpreted as a measure of this instability.

Specifically, consider figure $5 \mathrm{~A}$. Suppose that a household follows two distinct utility functions. These two utility functions give rise to two different demand functions: $d_{1}(p, I)$ and $d_{2}(p, I)$. Fix prices $p$ and suppose that we observe $x=d_{1}(p, I)$; see figure $5 A$. The second utility, on the other hand, would give demand $\hat{x}=d_{2}(p, I)$. Now, by continuity of demand, if we choose prices $p^{\prime}$ close to $p$ (as in the figure), then $x^{\prime}=d_{2}\left(p^{\prime}, I\right)$ is close to $\hat{x}$. But this implies a violation of WARP.

The money pump cost of the violation of WARP in figure $5 A$ is $\left(p^{\prime}-p\right) \cdot\left(x^{\prime}-x\right)$. We can look at the money pump cost for an arbitrarily small change in prices. In particular, fix a direction of change in price $\nabla p$ and consider an infinitesimal price change in $p$ in the direction of 

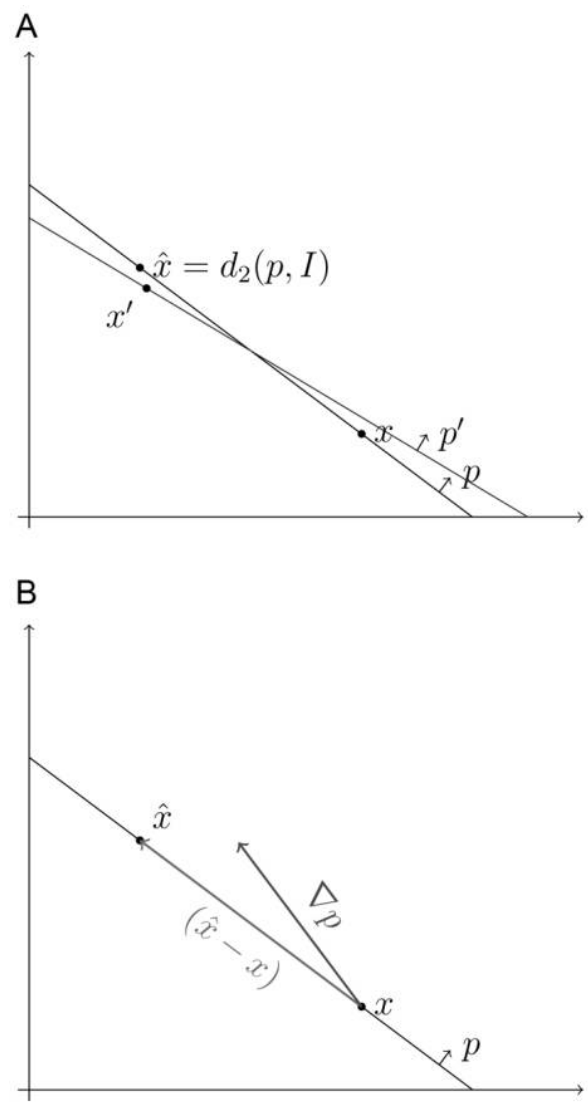

FIG. 5.-Unstable preferences. $A, x=d_{1}(p, I)$ and $x^{\prime}=d_{2}\left(p^{\prime}, I\right) . B, m p=\varepsilon \nabla p \cdot(\hat{x}-$ $x)$.

$\nabla p$. So $p^{\prime}=p+\varepsilon \nabla p$ for $\varepsilon>0$. As $\varepsilon$ shrinks to zero, $x^{\prime}$ converges to $\hat{x}$, so the money pump cost approaches $\varepsilon \nabla p \cdot(\hat{x}-x)$; see figure $5 B$. So a small price change gives an increase in the money pump cost as long as the change in prices forms an acute angle with the difference in the demand functions. Note also that a larger difference in demands results in a larger money pump cost for a given direction of change of prices.

Given this interpretation of the money pump cost as a measure of an agent's changes in preferences, we next look and see whether the MPI (money pump cost as the proportion of expenditure) reflects seasonal trends in demand for certain types of grocery items, because these trends may be attributable to changes in preferences over time. Specifically, we focus on the case of ice cream demand, for which the seasonal peak in demand occurs during the summer months. If this seasonality is in 
TABLE 4

No Evidence of Changing Preferences: Ice Cream versus Other Foods

\begin{tabular}{lcccc}
\hline \hline & Spring & Summer & Fall & Winter \\
\hline Spring & 1.3333 & .6944 & 1.0556 & .9375 \\
& $(1.90 \%)$ & $(1.84 \%)$ & $(2.00 \%)$ & $(2.16 \%)$ \\
Summer & $\ldots$ & 1.2000 & .8333 & 1.2292 \\
& $\ldots$ & $(2.13 \%)$ & $(1.65 \%)$ & $(1.52 \%)$ \\
Fall & $\ldots$ & $\ldots$ & 1.0667 & .9583 \\
& $\ldots$ & $\ldots$ & $(1.82 \%)$ & $(1.78 \%)$ \\
Winter & & & $\ldots$ & 1.6429 \\
& & & & $(1.87 \%)$ \\
\hline
\end{tabular}

NoTE.-Each cell contains the number of households that violate WARP between any two months, as classified by the season of the months. (Corresponding average MPIs for these violations are in parentheses.)

fact due to changing preferences, then we should expect to see larger MPIs in cycles involving peak and nonpeak periods than in cycles involving only nonpeak periods.

Such evidence is presented in table 4 . For this exercise, we aggregate consumption up to ice cream versus all other foods. For each pair of periods, we count the number of households violating WARP and compute the average of their median MPIs. Numbers (or parenthesized numbers) in the table are the numbers of households (or average MPIs), which are averaged over the pairs of periods falling into a corresponding pair of seasons.

Surprisingly, we find no evidence of seasonality. For instance, MPIs are 1.52 percent between the summer and winter months (a peak/ nonpeak comparison) versus 2.16 percent between the winter and spring months (two nonpeak periods). This suggests that, while seasonality may indeed be present, prices may also be moving in a fashion such that agents' resulting consumption choices do not violate GARP. ${ }^{10}$

More generally, we also repeated this exercise at the disaggregate level without aggregating across different goods. Overall, we found no systematic patterns between MPIs and the periods across which we were considering cycles. Again, this suggests that WARP violations are not related to time or seasonality trends. ${ }^{11}$

${ }^{10}$ Indeed, Chevalier, Kashyap, and Rossi (2003) provide evidence that prices on grocery items tend to be lower during peak demand periods for these items (see also Nevo and Hatzitaskos [2005] for further discussion). Such "countercyclical" price variation may mask any seasonal variation in preferences and lead to no violations of revealed preference.

${ }_{11}$ A similar empirical question of demand stability has been addressed in the agricultural economics literature using revealed preference methods, e.g., Chalfant and Alston (1988) and Jin and Koo (2003). 


\section{B. Power of GARP and the Bronars Index}

The vast majority of empirical studies of revealed preference find very few violations of GARP, a stylized fact that is referred to as the "low power" of GARP. To address this issue, "power indexes" have been developed to quantify the extent to which particular data sets may be useful for testing GARP. A power index seeks to measure the extent to which a collection of observed budget sets can detect violations of GARP. For example, if we have two budgets $B$ and $B^{\prime}$ that are nested (e.g., $B \subseteq$ $\left.B^{\prime}\right)$, then no choices by a consumer could reveal a violation of GARP. The earliest and most well-known power index is that of Bronars (1987). Specifically, the Bronars index measures the number of GARP violations under the behavioral assumption that consumers made purely random choices on their observed budget sets.

Typically, in cross-sectional data, income variability is much higher than price variability; as a result, budget sets tend to be nested, and no choices that exhaust the consumer's budget can violate GARP. In contrast, in our application using scanner data, we found that a large fraction of the households violate GARP. Nevertheless, we still computed the Bronars index for the budgets observed in our data. First, we draw random budget shares of goods from a continuous uniform distribution. The generated budget shares are then multiplied by observed total expenditure. We obtain the random consumption of each commodity by dividing its budget share by the price. We subsequently check GARP violation for each observation. ${ }^{12}$

We find, surprisingly, that the Bronars index indicates low power. We repeat this procedure 100 times and find that among 494 households there are, on average, only three to four violating GARP for each generated panel data set, much lower than the number of GARP violations observed in the actual choice data.

Our finding illustrates a basic problem with the Bronars index. A power index should reflect the probability that GARP is rejected when it should be rejected: but Bronars calculates the probability of rejecting GARP under purely random behavior. It is well known that random behavior can be very close to rational (see Becker 1962). One would instead want to measure the degree to which GARP is rejected under some alternative, clearly irrational, model of behavior. Bronars does not provide such a model; indeed, it seems very difficult to find an acceptable alternative benchmark to rationality under which to measure

${ }^{12}$ This computational method is the so-called method 2 in Andreoni et al. (2011). 
power; there is, in a sense, only one way to be rational but many more ways to be irrational. ${ }^{13}$

\section{Conclusion}

We present a new measure of the severity of a violation of GARP, the money pump index (MPI). The measure is based on the idea that a consumer who violates the axiom is subject to being exploited as a money pump. The MPI has a simple interpretation as the certain dollar amount that can be extracted from a consumer who behaves irrationally.

We carry out an empirical application using a scanner panel data set of households' food purchases at supermarkets. Almost all of the households in our sample violate the revealed preference axioms over the 2year sample period; on average, however, the MPI calculated for these violations is small, suggesting that the violations of revealed preference are not severe. This is supported by formal statistical testing, by which the hypothesis of consumer rationality cannot be rejected.

\section{References}

Afriat, Sidney N. 1967. "The Construction of Utility Functions from Expenditure Data." Internat. Econ. Rev. 8 (1): 67-77.

Andreoni, James, Ben Gillen, and William T. Harbaugh. 2011. "The Power of Revealed Preference Tests: Ex-Post Evaluation of Experimental Design.” Manuscript, Univ. California, San Diego.

Andreoni, James, and John Miller. 2002. "Giving According to GARP: An Experimental Test of the Consistency of Preferences for Altruism." Econometrica 70 (2): 737-53, http://www.jstor.org/stable/2692289.

Apesteguia, José, and Miguel A. Ballester. 2010. "A Measure of Rationality and Welfare." Manuscript, Univ. Pompeu Fabra.

Battalio, Raymond, John Kagel, Robin C. Winkler, Edwin B. Fischer, Robert Basmann, and Leonard Krasner. 1973. "A Test of Consumer Demand Theory Using Observations of Individual Consumer Purchases.” Western Econ. J. 11 (4): 411-28.

Beatty, Timothy K. M., and Ian A. Crawford. 2011. "How Demanding Is the Revealed Preference Approach to Demand?” A.E.R. 101 (6): 2782-95.

Becker, Gary S. 1962. "Irrational Behavior and Economic Theory." L.P.E. 70 (1): $1-13$.

Bell, David, and James Lattin. 1998. "Shopping Behavior and Consumer Preference for Store Price Format: Why 'Large Basket' Shoppers Prefer EDLP.” Marketing Sci. 17:68-88.

${ }^{13}$ There are alternative power indexes formulated by Famulari (1995) and by Andreoni et al. (2011). Andreoni et al.'s test, in particular, rests on a clever "reversion" of AEI to measure how far an observation that satisfies GARP is from not satisfying it. Since we are not mainly concerned with power, we have not calculated these alternative measures on our data set. They should be important to assess any empirical finding that fails to detect violations of GARP. 
Blundell, Richard. 1988. "Consumer Behaviour: Theory and Empirical Evidence-a Survey." Econ. I. 98 (389): 16-65.

Blundell, Richard, Martin Browning, and Ian Crawford. 2003. "Nonparametric Engel Curves and Revealed Preference." Econometrica 71 (1): 205-40.

Blundell, Richard, Xiaohong Chen, and Dennis Kristensen. 2007. "Seminonparametric IV Estimation of Shape-Invariant Engel Curves." Econometrica 75 (6): 1613-69.

Bronars, Stephen G. 1987. "The Power of Nonparametric Tests of Preference Maximization." Econometrica 55 (3): 693-98.

Chalfant, James A., and Julian Alston. 1988. "Accounting for Changes in Tastes." L.P.E. 96 (2): 391-410.

Chen, Keith, Venkat Lakshminarayanan, and Lauri Santos. 2006. "How Basic Are Behavioral Biases? Evidence from Capuchin Monkey Trading Behavior." I.P.E. 114:517-37.

Chevalier, Judith A., Anil K. Kashyap, and Peter E. Rossi. 2003. "Why Don't Prices Rise during Periods of Peak Demand? Evidence from Scanner Data." A.E.R. 93 (1): 15-37.

Choi, Syngjoo, Shachar Kariv, Wieland Müller, and Dan Silverman. 2011. "Who Is (More) Rational?” Manuscript, Univ. California, Berkeley.

Deaton, Angus S., and John Muellbauer. 1980. Economics and Consumer Behavior. Cambridge: Cambridge Univ. Press.

Famulari, Melissa. 1995. "A Household-Based, Nonparametric Test of Demand Theory." Rev. Econ. and Statis. 77 (2): 372-82.

Fevrier, Philippe, and Michael Visser. 2004. "A Study of Consumer Behavior Using Laboratory Data.” Experimental Econ. 7:93-114.

Gross, John. 1995. “Testing Data for Consistency with Revealed Preference.” Rev. Econ. and Statis. 77 (4): 701-10.

Harbaugh, William T., Kate Krause, and Timoth R. Berry. 2001. "GARP for Kids: On the Development of Rational Choice Behavior." A.E.R. 91 (5): 1539-45.

Hendel, Igal, and Aviv Nevo. 2006a. "Measuring the Implications of Sales and Consumer Stockpiling Behavior." Econometrica 74:1637-74.

- 2006b. "Sales and Consumer Inventory." Rand I. Econ. 37:543-61.

Heufer, Jan. 2008. "A Geometric Measure for the Violation of Utility Maximization.” Ruhr Economic Papers no. 069, RWI, Essen.

Hoderlein, Stefan, and Jörg Stoye. 2009. "Revealed Preferences in a Heterogeneous Population.” Manuscript, Brown Univ., Dept. Econ.

Jerison, David, and Michael Jerison. 2011. "Real Income Growth and Revealed Preference Inconsistency." Manuscript, Massachusetts Inst. Tech. and State Univ. New York Albany.

Jin, Hyun J., and Won W. Koo. 2003. "The Effect of the BSE Outbreak in Japan on Consumers' Preferences.” European Rev. Aoricultural Econ. 30:173-92.

Laibson, David, and Leeat Yariv. 2007. "Safety in Markets: An Impossibility Theorem for Dutch Books.” Manuscript, California Inst. Tech.

Mulligan, Casey. 1996. “A Logical Economist's Argument against Hyperbolic Discounting." Manuscript, Univ. Chicago.

Nevo, Aviv, and Konstantinos Hatzitaskos. 2005. "Why Does the Average Price of Tuna Fall during Lent?” Working Paper no. 11572, NBER, Cambridge, MA.

Rabin, Matthew. 2002. "A Perspective on Psychology and Economics." European Econ. Rev. 46 (4-5): 657-85.

Richter, Marcel K. 1966. "Revealed Preference Theory." Econometrica 34 (3): 63545 . 
Samuelson, Paul A. 1938. "A Note on the Pure Theory of Consumer's Behavior." Economica 5 (17): 61-71.

Shum, Matthew. 2004. "Does Advertising Overcome Brand Loyalty? Evidence from Breakfast Cereals." I. Econ. and Management Strategy 13:241-72.

Sippel, Reinhard. 1997. "An Experiment on the Pure Theory of Consumer's Behavior." Econ. I. 107:1431-44.

Varian, Hal R. 1982. "The Nonparametric Approach to Demand Analysis." Econometrica 50 (4): 945-74.

- 1985. "Non-parametric Analysis of Optimizing Behavior with Measurement Error." I. Econometrics 30:445-58.

. 1990. "Goodness-of-Fit in Optimizing Models." L. Econometrics 46 (1-2): $125-40$.

. 2006. "Revealed Preference." In Samuelsonian Economics and the TwentyFirst Century, edited by Michael Szenberg, Lall Ramrattan, and Aron A. Gottesman, 99-115. Oxford: Oxford Univ. Press. 